\title{
Improving Pneumococcal Vaccination Rates in Rheumatology Clinics
}

\author{
Julia G. Harris ${ }^{1}$
}

Rheumatology patients on immunosuppression are at increased risk of invasive pneumococcal disease. There is a multitude of literature highlighting the risk of pneumococcal infection, hospitalization, and even death in patients with systemic lupus erythematosus (SLE), in whom incidence of invasive pneumococcal infection is 13 times higher than the general population. ${ }^{1,2,3,4}$ Rheumatoid arthritis (RA) has also been deemed an at-risk condition and several other rheumatic diseases including polyarteritis nodosa, scleroderma, and Sjögren syndrome have increased hospitalization rates due to pneumococcal disease. ${ }^{2,3,4}$

The Centers for Disease Control and Prevention (CDC) recommends 2 different pneumococcal vaccinations-the 13 -valent pneumococcal conjugate vaccine (PCV13) and the 23-valent pneumococcal polysaccharide vaccine (PPSV23)for children aged 6 to 18 years and adults with immunocompromising conditions, which includes patients on iatrogenic immunosuppression. ${ }^{5,6}$ The European Alliance of Associations for Rheumatology vaccination recommendations state that pneumococcal vaccination should be strongly considered for the majority of patients with autoimmune inflammatory rheumatic diseases. ${ }^{7}$ The American College of Rheumatology RA treatment guidelines recommend pneumococcal vaccines for all treatment groups. ${ }^{8}$ Receipt of pneumococcal vaccines by adults and children with chronic inflammatory diseases on immunosuppression is also recommended by the Infectious Diseases Society of America.

Despite the risk of invasive pneumococcal disease in many rheumatology patients and the pneumococcal vaccination recommendations summarized above, pneumococcal vaccination rates in this population overall are suboptimal, prompting

${ }^{1}$ J.G. Harris, MD, Associate Professor of Pediatrics, Department of Pediatrics, Children's Mercy Kansas City, Kansas City, Missouri, USA.

The author declares no conflicts of interest.

Address correspondence to Dr. J.G. Harris, Division of Rheumatology,

Children's Mercy Kansas City, 2401 Gillham Road, Kansas City, MO

64108,USA.Email:jgharris@cmh.edu. many centers to engage in quality improvement efforts to increase their vaccination rates. ${ }^{10-19}$

In this issue of The Journal of Rheumatology, Sheth and colleagues highlight their quality improvement project to improve pneumococcal vaccination rates in 13 rheumatology clinics using best practice alerts in the electronic health record (EHR). ${ }^{19}$ Their project started in 2013, with phase I providing PPSV23 to patients with RA on immunosuppression. Over a 1.5-year period, PPSV23 rates increased from $27.9 \%$ to $61.5 \%$. Phase II of the project expanded the target population to any patient on immunosuppressive therapy and patients were given both PCV13 and PPSV23. Vaccination rates during this 1.5-year phase also increased from $49.6 \%$ to $77 \%$ for patients having received a PCV13 and/or PPSV23. The authors highlight that their primary intervention in this quality improvement project was a best practice alert in the EHR that was developed based on CDC guidelines. The project also involved education of physicians, staff, and patients; regular feedback to physicians and staff on vaccination rates; and a survey of physicians and staff that concluded that the best practice alert did not increase work burden.

Among the many strengths of the paper by Sheth, et al, the most notable is the sheer number of eligible patients includedover 5000 for phase I and 26,717 for phase II-spread across 13 clinics. ${ }^{19}$ It is quite impressive that almost 24,000 patients received a PCV13 and/or PPSV23 during this project. The number of clinics involved supports the adoptability of the project's intervention to other clinics or institutions. The authors highlight how the ancillary staff involvement, specifically the clinics' medical assistants, contributed to the success of their project and led to less physician burden. Other pneumococcal vaccine quality improvement efforts have used a nurse-driven model. ${ }^{14,16}$ I agree that it is ideal to have engagement of a multidisciplinary team for any quality improvement project. The approach taken in this study of administering pneumococcal vaccines in the rheumatology clinic at the time of vaccine recommendation is ideal. Murray and colleagues previously demon-

\section{See Improving pneumococcal vaccination rates, page 1472}


strated that interventions focused on communicating with primary care physicians to administer the vaccine and providing patient education failed to increase vaccination rates. ${ }^{18}$ The authors also performed a logistic regression analysis and found positive associations between getting at least 1 pneumococcal vaccine with age $\geq 65$ years and receiving care at an academic center. ${ }^{19}$ Another study found that the volume of patients with SLE seen by a rheumatologist is strongly associated with receipt of PPSV23. ${ }^{20}$

The best practice alert used by Sheth and colleagues was not just a simple alert but rather had embedded clinical decision support; this allowed clinicians to reference vaccine eligibility based on CDC guidelines, order pneumococcal vaccines, and document refusal or deferral reasons. ${ }^{19} \mathrm{~A}$ recent systematic review and metaanalysis of 108 studies found that clinical decision support in most projects led to small-to-moderate improvements in the intended care. ${ }^{21}$ Best practice alerts have led to improved vaccination rates in rheumatology and other specialties ${ }^{16,22,23}$; one of these studies, by Ledwich and colleagues, that led to improved pneumococcal vaccination rates from $19 \%$ to $41 \%$ also used a best practice alert in the EHR. ${ }^{16}$ However, success with best practice alerts is not universal, as a pneumococcal best practice alert for high-risk adults 19 to 64 years old in a primary care setting did not lead to an increased vaccination rate..$^{24}$ Another major intervention to improve pneumococcal vaccination rates in rheumatology patients includes previsit planning, which involves review of the EHR prior to the clinic visit. ${ }^{11,12,17}$ Point-of-care paper reminders have also been used, and this was the major intervention that led to improved pneumococcal vaccination rates in immunosuppressed rheumatology patients in a study by Desai and colleagues. ${ }^{15}$ Reviewing vaccination rate progress during the intervention phase was also common practice for many quality improvement projects. ${ }^{12,17,19}$

Despite the strengths of the manuscript by Sheth, et al, there are several limitations. ${ }^{19}$ First, the postintervention data does not independently depict whether patients are completely up to date on pneumococcal vaccines, namely if they received both PCV13 and PPSV23, rather than just 1 pneumococcal vaccine. It is also unclear why the authors chose to focus solely on PPSV23 for phase I of the study despite the CDC guidelines having been published prior to the start of this project recommending PCV13 followed by PPSV23. ${ }^{6}$ Further, if PPSV23 is given prior to PCV13 in an adult, 1 year is needed between the vaccinations (for children, it is $\geq 8 \mathrm{wks}$ ) rather than the 8 weeks needed between the vaccines in the preferred PCV13-PPSV23 sequence. ${ }^{5,6}$ The best practice alert for the authors was inactive for 6 months after a vaccine was ordered; this does not align with the aforementioned CDC vaccine timing recommendations. ${ }^{5,6}$

Despite the increase in vaccination rates for Sheth and colleagues, the best practice alert led to no action in $56.5 \%$ of encounters. ${ }^{19}$ In the spirit of continuous improvement, this ongoing gap between the actual and ideal states creates an opportunity for the authors to investigate further and address any barriers. Although the authors suggest that provider factors contributed to this alert "noncompliance," this assumption may underestimate the complexity of barriers to improved performance.

This quality improvement project would be enhanced by having a more complete set of quality measures: outcome, process, and balancing measures..$^{25}$ For example, best practice alert "compliance" could be a process measure that is tracked over time. A balancing measure, which is used to detect unintended consequences, could have been the time the medical assistant spent with the patient. Last, displaying data from a quality improvement project on a control chart is ideal to look for nonrandom variation, ${ }^{25}$ and this visual tool has been used in many pneumococcal vaccine improvement projects in rheumatology. ${ }^{11,12,13}$

In conclusion, Sheth and colleagues should be commended for their success in vaccinating thousands of patients to decrease their risk of invasive pneumococcal disease. ${ }^{19} \mathrm{I}$ hope that other centers will follow suit in establishing pneumococcal vaccination improvement initiatives to decrease morbidity and mortality from this serious infection. Through education, utilization of quality improvement methodology, and sharing of best practices, the rheumatology field can improve the care of our immunosuppressed patients.

\section{REFERENCES}

1. Luijten RK, Cuppen BV, Bijlsma JW, Derksen RH. Serious infections in systemic lupus erythematosus with a focus on pneumococcal infections. Lupus 2014;23:1512-6.

2. Furer V, Rondaan C, Heijstek M, van Assen S, Bijl M, Agmon-Levin $\mathrm{N}$, et al. Incidence and prevalence of vaccine preventable infections in adult patients with autoimmune inflammatory rheumatic diseases (AIIRD): a systemic literature review informing the 2019 update of the EULAR recommendations for vaccination in adult patients with AIIRD. RMD Open 2019;5:e001041.

3. Shea KM, Edelsberg J, Weycker D, Farkouh RA, Strutton DR, Pelton SI. Rates of pneumococcal disease in adults with chronic medical conditions. Open Forum Infect Dis 2014; 1: ofu024.

4. Wotton CJ, Goldacre MJ. Risk of invasive pneumococcal disease in people admitted to hospital with selected immune-mediated diseases: record linkage cohort analyses. J Epidemiol Community Health 2012;66:1177-81.

5. Centers for Disease Control and Prevention (CDC). Use of 13-valent pneumococcal conjugate vaccine and 23-valent pneumococcal polysaccharide vaccine among children aged 6-18 years with immunocompromising conditions: recommendations of the Advisory Committee on Immunization Practices (ACIP). MMWR Morb Mortal Wkly Rep 2013;62:521-4.

6. Centers for Disease Control and Prevention (CDC). Use of 13-valent pneumococcal conjugate vaccine and 23 -valent pneumococcal polysaccharide vaccine for adults with immunocompromising conditions: recommendations of the Advisory Committee on Immunization Practices (ACIP). MMWR Morb Mortal Wkly Rep 2012;61:816-9.

7. Furer V, Rondaan C, Heijstek MW, Agmon-Levin N, van Assen S, Bijl M, et al. 2019 update of EULAR recommendations for vaccination in adult patients with autoimmune inflammatory rheumatic diseases. Ann Rheum Dis 2020;79:39-52.

8. Singh JA, Saag KG, Bridges L, Akl EA, Bannuru RR, Sullivan MC, et al. 2015 American College of Rheumatology guideline for the treatment of rheumatoid arthritis. Arthritis Care Res 2016;68:1-26.

9. Rubin LG, Levin MJ, Ljungman P, Davies EG, Avery R, Tomblyn 
M, et al; Infectious Diseases Society of America. 2013 IDSA clinical practice guideline for vaccination of the immunocompromised host. Clin Infect Dis 2014;58:309-18.

10. Subesinghe $S$, Rutherford AI, Ibrahim F, Harris H, Galloway J. A large two-centre study in to rates of influenza and pneumococcal vaccination and infection burden in rheumatoid arthritis in the UK. BMC Musculoskelet Disord 2016;17:322.

11. Sivaraman V, Wise KA, Cotton W, Barbar-Smiley F, AlAhmed $\mathrm{O}, \mathrm{MacD}$ onald D, et al. Previsit planning improves pneumococcal vaccination rates in childhood-onset SLE. Pediatrics 2020;145: e20183141.

12. Harris JG, Maletta KI, Ren B, Olson JC. Improving pneumococcal vaccination in pediatric rheumatology patients. Pediatrics 2015;136:e681-6.

13. Wilson J, Swee M, Mosher H, Scott-Cawiezell J, Levins L, Fort K, et al. Using Lean Six Sigma to improve pneumococcal vaccination rates in a Veterans Affairs Rheumatology Clinic. J Healthc Qual 2020; $42: 166-74$.

14. Pennant KN, Costa JJ, Fuhlbrigge AL, Sax PE, Szent-Gyorgyi LE, Coblyn J, et al. Improving influenza and pneumococcal vaccination rates in ambulatory specialty practices. Open Forum Infect Dis 2015;2:ofv119.

15. Desai SP, Lu B, Szent-Gyorgyi LE, Bogdanova AA, Turchin A, Weinblatt $M$, et al. Increasing pneumococcal vaccination for immunosuppressed patients: a cluster quality improvement trial. Arthritis Rheum 2013;65:39-47.

16. Ledwich LJ, Harrington TM, Ayoub WT, Sartorius JA, Newman ED. Improved influenza and pneumococcal vaccinations in rheumatology patients taking immunosuppressants using an electronic health record best practice alert. Arthritis Rheum 2009;61:1505-10.
17. Garg S, Tsagaris K, Cozmuta R, Lipson A. Improving the combination pneumococcal vaccination rate in systemic lupus erythematosus patients at an adult rheumatology practice. J Rheumatol 2018;45:1656-62.

18. Murray K, Low C, O’Rourke A, Young F, Callanan I, Feeney E, et al. A quality improvement intervention failed to significantly increase pneumococcal and influenza vaccination rates in immunosuppressed inflammatory arthritis patients. Clin Rheumatol 2020;39:747-54.

19. Sheth H, Grimes V, Rudge D, Ayers B, Moreland L, Fischer G, et al. Improving pneumococcal vaccination rates in rheumatology patients by using best practice alerts in the electronic health records. J Rheumatol 2021;48:1472-9.

20. Arora S, Nika A, Sequeira W, Block JA, Jolly M. Pneumococcal vaccination among lupus patients: who are the recipients? J Clin Rheumatol 2020 Aug 14 (E-pub ahead of print).

21. Kwan JL, Lo L, Ferguson J, Goldberg H, Diaz-Martinez JP, Tomlinson G, et al. Computerised clinical decision support systems and absolute improvement in care: meta-analysis of controlled clinical trials. BMJ 2020;370:m3216.

22. Malone K, Clark S, Palmer JA, Lopez S, Pradhan M, Furth S, et al. A quality improvement initiative to increase pneumococcal vaccination coverage among children after kidney transplant. Pediatr Transplantation 2016;20:783-9.

23. Klatt T, Hopp E. Effect of a best-practice alert on the rate of influenza vaccination of pregnant women. Obstet Gynecol 2012;119:301-5.

24. McAdam-Marx C, Tak C, Petigara T, Jones NW, Yoo M, Struwe Briley M, et al. Impact of a guideline-based best practice alert on pneumococcal vaccination rates in adults in a primary care setting. BMC Health Services Research 2019;19:474.

25. Provost LP, Murray SK. The Health care data guide: learning from data for improvement. San Francisco: Josey-Bass; 2011. 